ORIGINAL ARTICLE

\title{
Anatomical distribution of colorectal cancer over a 10 year period in a district general hospital: is there a true "rightward shift"?
}

\author{
D Gomez, Z Dalal, E Raw, C Roberts, P J Lyndon
}

Postgrad Med J 2004;80:667-669. doi: 10.1136/pgmj.2004.020198

\begin{abstract}
Introduction: Recent studies of patients with colorectal cancer have suggested a shift towards the proximal colon and an increase in the incidence of right sided colon cancer. This study aimed to determine the anatomical distribution of colorectal cancer over a 10 year period in a district general hospital.

Methods: Records of patients diagnosed with primary colorectal cancer from 1993 to 2002 were reviewed for demographic data, histology subtype, and anatomical location of the tumour. Tumours located at and proximal to the splenic flexure were defined as right sided cancer and tumours arising distal to the splenic flexure were defined as left sided cancer.

Results: A total of 763 patients were included in the study, of whom all had adenocarcinoma and $99 \%$ were white. Sixty nine percent of cancers were left sided and $31 \%$ were right sided. Although there was a $4 \%$ increase in the proportion of right sided cancers, there was no statistically significant increase using logistic regression analysis. Mann-Whitney $U$ test revealed no significant difference in age at diagnosis between the right and left sided cancers. Although a higher proportion of females were diagnosed with right sided cancer compared with left sided cancer, this was not statistically significant.

Conclusion: The anatomical distribution of colorectal cancer has been fairly stable at this hospital with no evidence of a shift towards the proximal colon. No differences were identified in the tumour distribution with respect to gender and age at diagnosis. Our findings support the initial application of flexible sigmoidoscopy for investigating patients with suspected colorectal malignancy and follow up colonoscopy for selected patients to exclude right sided pathology.
\end{abstract}

See end of article for authors' affiliations

Correspondence to:

MrP J Lyndon, Department of Surgery, Dewsbury Health Care NHS Trust Halifax Road, Dewsbury WF13 4HS, UK; christine. ledger@dhc-tr.northy.nhs. uk

Submitted 9 February 2004 Accepted 18 March 2004
$T$ he incidence of colorectal cancer throughout the UK has been rising over the last 30 years. ${ }^{1}$ Various studies have reported a shift in the anatomical distribution of colorectal cancer towards the proximal colon. ${ }^{2-11}$ It is important to establish whether this reported change in anatomical distribution is genuine as this could have a major implication on current referral practices and the methods used for investigating patients with suspected colorectal malignancy. To be able to assess our current practices in investigating these patients, it is important to appreciate variation in the anatomical distribution of colorectal cancer and whether this is indeed changing over a period of time.

In this study, the hospital's pathology database has been used to identify patients with a histological diagnosis of colorectal cancer with analysis of the site of the cancer in patients referred to the Dewsbury Healthcare NHS Trust over a 10 year period.

\section{METHODS}

Patients diagnosed with primary colorectal cancer at the Dewsbury Health Care NHS Trust during the 10 year period, from 1993 to 2002, were identified using the pathology computer coding system (Snomed). This search was considered reliable, as it was able to identify any patient with the histological diagnosis of colorectal cancer. The histology report, surgical reports, and case notes of each patient were reviewed for age at diagnosis, gender, ethnicity, histology subtype, year of diagnosis, and anatomical location of the tumour. Colonic tumours located at the caecum, ascending colon, hepatic flexure, transverse colon, and splenic flexure were defined as right sided colon cancer. Tumours located at the descending colon, sigmoid, and rectum were defined as left sided colorectal cancer.
The anatomical distribution of the cancers was determined for each year during the 10 year period. Ethnicity was defined as white, Afro-Caribbean, Asian, Oriental, and others. Continuous data were assessed for normality by a one sample Kolmogorov-Smirnov test. Non-parametric data are presented as mean (SD), and categorical data as both frequency and proportion (\%). Logistic regression analysis was performed to determine whether there was a statistically significant change in tumour distribution over the study period. Mann-Whitney $U$ test was used to assess for a difference in age at diagnosis between the right and left sided cancers. The association between gender and tumour distribution was assessed using a $\chi^{2}$ test. All statistical analyses were performed using the Statistical Package for the Social Sciences for Windows version 11.5 (SPSS Inc, Chicago, Ill, USA), and statistical significance was taken at the $5 \%$ level.

\section{RESULTS}

During the study period, a total of 771 cases of primary colorectal cancer were identified with eight cases having both left and right sided synchronous colorectal cancers. These eight cases were excluded from our study. From the total of 763 cases studied, $99 \%$ were white and the male to female ratio was 14:11. The histology subtype of all primary colorectal cancers during this period was adenocarcinoma. The mean (SD) age of patients in this study was 70 (11) years.

Using the anatomical definition proposed, a total of $31 \%$ of cancers were right sided over the 10 year study period. Figure 1 demonstrates the changes in anatomical distribution of colorectal cancer for the patient population sampled over the 10 years. During the study period, the percentage 
proportion of right sided colon cancer rose from $25 \%$ in year 1993 to $29 \%$ in year 2002. The highest proportion of right sided colon cancers recorded was $40 \%$ in year 2001. To determine whether there was a significant difference in the proportion of right sided colon cancers during the study period, a logistic regression analysis was used. This test indicated that the odds on right sided cancers are increasing by a factor of 1.026 (95\% confidence interval 0.974 to 1.081 ) per year. The result of this analysis revealed no statistically significant difference between the time periods in the proportion of right sided colon cancers $\left(\chi^{2}=0.931, \mathrm{df}=1\right.$, $\mathrm{p}=0.335)$. Hence, the apparent increase in the proportion of right sided colon cancers over the study period is not statistically significant.

Analysis of age at diagnosis when compared to tumour distribution revealed no difference. The mean (SD) age at diagnosis of patients with right sided cancers was 71 (11) years. In the group of patients with left sided cancers, the median (SD) age at diagnosis was 70 (12) years. MannWhitney $U$ test was performed to determine whether there was a significant difference in age at diagnosis between the right and left sided cancers. The result of this analysis was not statistically significant $(\mathrm{p}=0.461)$.

There were modest differences in the anatomical distribution between the sexes with a slightly higher proportion of females diagnosed with right sided disease. In patients with right sided colon cancer, $48 \%$ were female compared to $41 \%$ with left sided colorectal cancer. In the group with left sided colorectal cancer, $59 \%$ were male compared to $52 \%$ of males with cancers on the right side. Analysis by $\chi^{2}$ revealed no statistically significant association between gender and anatomical distribution of colorectal cancer during the 10 year period $\left(\chi^{2}=3.434, \mathrm{df}=1, \mathrm{p}=0.064\right)$. Hence, the apparent higher proportion of females with right sided colon cancer over the 10 year period is not statistically significant. Details of our study sample and principal results are summarised in table 1. A separate analysis was not performed to compare the difference between ethnic backgrounds because of the small numbers in groups of nonwhite patients.

\section{DISCUSSION}

With the incidence of colorectal cancer steadily rising, ${ }^{1}$ it is essential that appropriate diagnostic techniques are applied to reduce its associated morbidity and mortality. The present study, which covered a period of 10 years, from 1993 to 2002, investigated the changes in the anatomical distribution of colorectal cancer in a district general hospital over the period studied. Our data revealed that $31 \%$ of all colorectal cancers

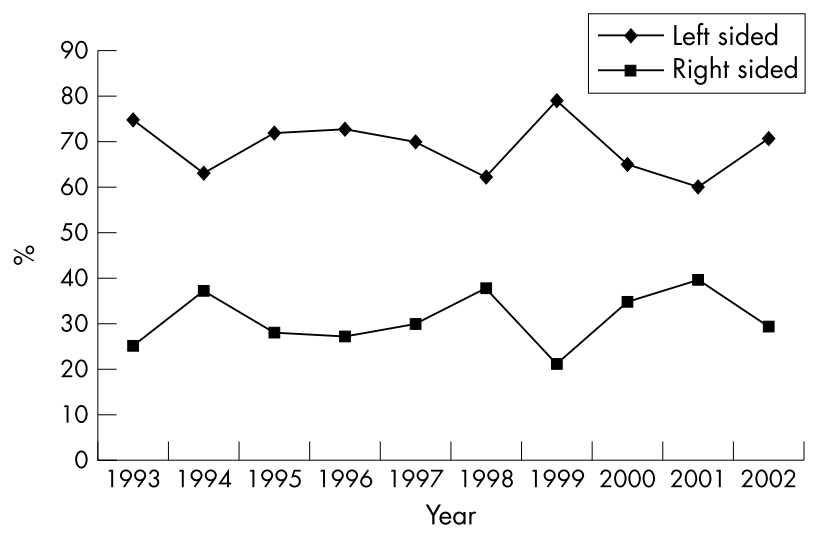

Figure 1 Changes in the anatomical distribution of colorectal cancer for the patient population sampled over the 10 year study period expressed as a percentage (right sided: $n=238$, left sided: $n=525$ ).
Table 1 Demographics of the study population for right and left sided disease

\begin{tabular}{llll}
\hline & $\begin{array}{l}\text { Right sided } \\
\text { (n=238; 31\%) }\end{array}$ & $\begin{array}{l}\text { Left sided } \\
(\mathbf{n}=525 ; 69 \%)\end{array}$ & $\mathbf{p}$ Value \\
\hline $\begin{array}{l}\text { Mean (SD) age at } \\
\text { diagnosis }\end{array}$ & $71(11)$ & $70(12)$ & 0.461 \\
$\begin{array}{l}\text { Male sex: total cases (\%) } \\
\text { 123 (52) }\end{array}$ & $309(59)$ & 0.064 \\
\hline
\end{tabular}

were right sided and there was a $4 \%$ increase in the proportion of right sided colon cancers. However, this increase was not statistically significant. Some authors have described a change in the anatomical distribution of colorectal cancers towards the proximal location, ${ }^{2-9}$ while others have not confirmed this shift. ${ }^{10-12}$ In this study, we were unable to establish a significant increase in the proportion of right sided colon cancer. The question, however, remains: Is there a true shift towards proximal colonic tumours? The majority of North American studies have reported a left to right shift in colorectal cancer. ${ }^{2-4} 689$ However, our study did not reveal such a change, suggesting that this shift may be less prevalent in the UK. Waldron and Donovan revealed that $23 \%$ of colorectal tumours were right sided (defined as tumours arising from the caecum, ascending colon, and hepatic flexure) during their 10 year study period in Birmingham. ${ }^{13}$ A 30 year study in Dublin revealed that approximately $28 \%$ of colorectal tumours were right sided as defined by us. ${ }^{11}$ A recent study on the current trends of colorectal cancer in England and Wales concluded that approximately $29 \%$ of colorectal cancers were right sided by applying our definition from 1971 to $1994 .{ }^{14}$ This suggests that our findings are representative of the UK and Ireland.

Our study revealed no statistical difference between the age at diagnosis in right and left sided cancers. This contrasts with previous studies which have suggested that right sided colon cancers are diagnosed in the older age groups compared with left sided colorectal cancers. ${ }^{4}{ }^{11}$ Nevertheless, Adloff et al reported similar distribution between left and right sided disease in all age groups, ${ }^{15}$ and another study reported a predominance of right sided disease in the younger age groups. ${ }^{16}$ The findings from our study suggest that colorectal cancer is a disease mainly diagnosed in the elderly irrespective of the tumour location.

This study demonstrated no significant differences in gender between right and left sided cancers. Previous studies have shown that proximal cancers were more often observed in females. ${ }^{3}{ }^{11} 12$ Recent epidemiology studies in the UK and North America have not confirmed this association. ${ }^{8}{ }^{14}$ In this study, there was a moderately higher proportion of females who were diagnosed with right sided colon cancer compared with cancers that occurred on the left side. However, this was not statistically significant. Differences observed in tumour distribution with regards to age at diagnosis and gender has lead to the hypothesis that various genetic and environmental factors could be implicated in the aetiology of these two different cancer types. ${ }^{17}$

This study shows that there has been no significant change in the anatomical distribution of colorectal cancer in the Dewsbury district over the last 10 years. More importantly, the proportion of right sided colon cancer in this district has been consistent and is representative of the UK and Ireland. We also observed that there is no significant difference in anatomical distribution of colorectal cancer when compared to age at diagnosis and gender. The result of this study has important implications for the selection of the most appropriate investigation for patients with suspected colorectal cancer. Given the distribution of the majority of colorectal 
cancers, and the new range of $90 \mathrm{~cm}$ flexible sigmoidoscopes, sigmoidoscopy is an appropriate first line investigation for patients with bowel symptoms suggestive of colorectal malignancy with no age or gender bias. Thereafter, this can be supplemented by the selective use of colonoscopy based on clinical features and sigmoidoscopy findings.

\section{CONCLUSION}

The anatomical distribution of colorectal cancer has significant clinical implications for investigating patients with suspected colorectal malignancy. This study has shown that the proportion of right sided colon cancer has been stable with no evidence of a rightward shift over the last 10 years. Our findings support the application of flexible sigmoidoscopy as a first line investigation for patients with suspected colorectal cancer and a follow up colonoscopy for selected patients to exclude right sided pathology.

\section{Authors' affiliations}

D Gomez, Z Dalal, E Raw, C Roberts, P J Lyndon, Department of Surgery, Dewsbury Health Care NHS Trust, Dewsbury, UK

\section{REFERENCES}

1 Wakefield SE, Wheeler DW, Mortensen NJ, et al. The incidence and Dukes' staging of colorectal cancer over 3 decades. Eur J Surg Oncol 1998:24:525-7.

2 Axtell LM, Chiazze L. Changing relative frequency of cancers of the colon and rectum in United States. Cancer 1966;19:750-4.
3 Abrams JS, Reines HD. Increasing incidence of right sided lesions in colorectal cancer. Am J Surg 1979;137:522-6.

4 Schub R, Steinheber FU. Rightward shift of colon cancer. A feature of the aging gut. J Clin Gastroenterol 1986;8:630-4.

5 Kee F, Wilson RH, Gilliland R, et al. Changing site in the distribution of colorectal cancer. BMJ 1992;305:158.

6 Devesa SS, Chow WH. Variation in colorectal cancer incidence in the United States by the subsite of origin. Cancer 1993;71:3819-25.

7 Obrand DI, Gordon PH. Continued change in the distribution of colorectal carcinoma. Br J Surg 1998;85:246-8.

8 Cucino C, Buchner AM, Sonnenberg A. Continued rightward shift of colorectal cancer. Dis Colon Rectum 2002;45:1035-40.

9 Rabeneck L, Davila JA, El-Serag HB. Is there a true "shiff" to the right colon in the incidence of colorectal cancer? Am J Gastroenterol 2003;98:1400-9.

10 Stewart RJ, Stewart AW, Turnbull PRG, et al. Sex differences in subsite incidence of large bowel cancer. J Am Soc Colon Rectal Surg 1983;26:658-60.

11 Crerand S, Freely TM, Waldron RP, et al. Colorectal carcinoma over 30 years at one hospital: no evidence of a shift to the right. Int J Colorectal Dis 1991:6:184-7.

12 Sharma VK, Vasudeva R, Howden CW. Changes in colorectal cancer over a 15-year period in a single United States city. Am J Gastroenterol 2000;85:3615-9.

13 Waldron RP, Donovan IA. Mortality in patients with obstructing colorectal cancer. Ann R Coll Surg Engl 1986;68:219-21.

14 Hayne D, Brown RS, McCormack M, et al. Current trends in colorectal cancer: site, incidence, mortality and survival in England and Wales. Clin Oncol 2001;13:448-52

15 Adloff M, Arnaud JP, Schloegel M, et al. Colorectal cancer in patients under 40 years of age. Dis Colon Rectum 1986;29:322-5.

16 Simstein NI, Kovalcik PJ, Cross GH. Colorectal carcinoma in patients less than 40 years old. Dis Colon Rectum 1978;21:169-71.

17 Ponz DLM, Sacchetti C, Sassatelli R, et al. Evidence for the existence of different types of large bowel tumour: suggestions from clinical data of a population based registry. J Surg Oncol 1990;44:35-43. 